\title{
Frequency of EGFR Mutations in Non-small Cell Lung Cancer Patients: Screening Data from West Siberia
}

\author{
Polina Gervas $^{1 * \&}$, Anna Ivanova ${ }^{1 \&}$, Nikolay Vasiliev ${ }^{1}$, Olga Ananina ${ }^{1}$, Olga \\ Zharkova $^{2}$, Olga Rogovieva ${ }^{2}$, Natalia Verzhbitskaya ${ }^{2}$, Ivan Didichuk ${ }^{3}$, Olga \\ Cheremisina $^{1}$, Natalia Popova ${ }^{1}$, Victor Goldberg ${ }^{1}$, Evgeny Cherdyntsev $^{4}$, Evgeny \\ Choynzonov $^{1}$, Nadezda Cherdyntseva ${ }^{1,5}$
}

\begin{abstract}
Background: Incorporation of molecular analysis of the epidermal growth factor receptor (EGFR) gene into routine clinical practice has shown great promise to provide personalized therapy of the non-small cell lung cancer (NSCLC) in the developed world. However, the genetic testing of EGFR mutations has not yet become routine clinical practice in territories remote from the central regions of Russia. Therefore, we aimed to study the frequency of major types of activating mutations of the EGFR gene in NSCLC patients residing in West Siberia. Materials and Methods: We examined EGFR mutations in exons 19 and 21 in 147 NSCLC patients (excluding squamous cell lung carcinomas) by real time polymerase chain reaction. Results: EGFR mutations were detected in 28 of the $147(19 \%)$ patients. There were $19(13 \%)$ cases with mutations in exon 19 and 9 cases $(6 \%)$ in exon 21. Mutations were more frequently observed in women $(42 \%, p=0.000)$ than in men $(1 \%)$. A significantly higher incidence of EGFR mutations was observed in bronchioloalveolar carcinomas $(28 \%, p=0.019)$ and in adenocarcinomas $(21 \%, p=0.024)$ than in large cell carcinomas, mixed adenocarcinomas, and NOS (4\%). The EGFR mutation rate was much higher in never-smokers than in smokers: $38 \% \mathrm{vs.3 \%}(\mathrm{p}=\mathbf{0 . 0 0 0})$. The frequency of EGFR mutations in the Kemerovo and Tomsk regions was $19 \%$. Conclusions: The incorporation of molecular analysis of the EGFR gene into routine clinical practice will allow clinicians to provide personalised therapy, resulting in a significant increase in survival rates and improvement in life quality of advanced NSCLC patients.
\end{abstract}

Keywords: Non-small cell lung cancer - epidermal growth factor receptor - Western Siberia

Asian Pac J Cancer Prev, 16 (2), 689-692

\section{Introduction}

Lung cancer remains one of the most common cancers in the world and the leading cause of cancer-related death (Cherdyntseva et al., 2010; Jemal et al., 2010). The identification of driver oncogene mutations, such as activating mutations in the epidermal growth factor receptor gene (EGFR), is a promising approach that can improve treatment outcomes in patients with non-small cell lung cancer (NSCLC). Over the last decade, two small molecules, the selective and reversible EGFR-tyrosine kinase inhibitors (TKIs) gefitinib and erlotinib, have been extensively investigated for the treatment of NSCLC patients. In 2004, several studies demonstrated a significant benefit of tyrosine kinase inhibitor treatment compared to conventional chemotherapy for NSCLC patients harbouring EGFR-activating mutations (Maemondo et al., 2010). EGFR mutations appear to be concentrated in exons 18 to 21 of EGFR. The most common mutation was found in two hotspots: a short in-frame deletion in exon 19 and a point mutation at codon 858 in exon 21 L858R. In patients with NSCLC harbouring EGFR mutations, first-line therapy with gefitinib was associated with longer progression-free survival, a higher objective response rate, more favourable toxicity profile, and better quality of life compared to conventional chemotherapy. Gefitinib currently represents the best first-line therapy for appropriately selected subgroup of patients (Maemondo et al., 2010; Gridelli et al., 2011). However, the genetic testing of EGFR mutations has not yet become routine clinical practice in territories remote from the central regions of Russia (Imianitov et al., 2008 ; Ponomareva et al., 2011). To our knowledge, there are only two Russian reports, published in conference proceedings dedicated to the genetic testing of EGFR mutation in the region of West Siberia. Therefore, we aimed to study the frequency 
Table 1. EGFR Gene Mutations and Patients' Clinicopathological Characteristics

\begin{tabular}{llcccc}
\hline Variables & & \multicolumn{2}{c}{ EGFR Gene Mutation Status } & p value \\
& & $\begin{array}{c}\text { All patients } \\
(\mathrm{n}=147)\end{array}$ & $\begin{array}{c}\text { Mutated } \\
(\mathrm{n}=28)\end{array}$ & $\begin{array}{c}\text { Wildtype } \\
(\mathrm{n}=119)\end{array}$ \\
\hline Gender & male & 82 & $1(1 \%)$ & $81(99 \%)$ & 0.00 \\
\multirow{5}{*}{ Tumor histology } & female & 65 & $27(42 \%)$ & $38(58 \%)$ & $0.024 *$ \\
& adenocarcinoma & 95 & $20(21 \%)$ & $75(79 \%)$ & $0.019 * *$ \\
& bronchioloalveolar carcinoma & 25 & $7(28 \%)$ & $18(72 \%)$ & \\
& large cell carcinoma & 8 & 0 & $8(100 \%)$ & \\
& mixed adenocarcinoma & 6 & 0 & $6(100 \%)$ & \\
Smoking status & NOS (non other specified) & 13 & $1(8 \%)$ & $12(92 \%)$ & \\
\multirow{3}{*}{ Regions of West Siberia } & Never-smoker & 69 & $26(38 \%)$ & $43(62 \%)$ & 0.00 \\
& Former/Current smoker & 78 & $2(3 \%)$ & $76(97 \%)$ & \\
& Kemerovo & 90 & $17(19 \%)$ & $73(81 \%)$ & \\
\hline
\end{tabular}

*adenocarcinoma vs. combined group (large cell carcinoma, mixed adenocarcinoma, NOS); **bronchioloalveolar carcinoma vs combined group (large cell carcinoma, mixed adenocarcinoma, NOS)

of major types of activating mutations of the EGFR gene in NSCLC patients residing in West Siberia.

\section{Materials and Methods}

\section{Eligibility criteria}

For molecular screening, we selected the following histological types of NSCLC: adenocarcinoma, bronchioloalveolar carcinoma, large cell carcinoma, poorly differentiated carcinoma, adenosquamous carcinoma (mixed adenocarcinoma), and NSCLC-NOS (not otherwise specified).

\section{Patients}

The study began in February 2012. A total of 147 patients treated at the Clinic of Cancer Research Institute (Tomsk) and Regional Oncology Centers of Tomsk and Kemerovo were included in the study. Smoking history status was based on self-reported questionnaires. All patients were of Slavic origin (Russian, Ukrainian and Belarusian). Tumours were histologically confirmed and staged in accordance with the tumour-node-metastasis classification of malignant tumours (T1-3N0-3M0-M1). Written informed consent was obtained from each patient.

\section{Biomarker analysis}

Tumour samples were obtained from archived formalin-fixed paraffin-embedded tissue blocks, and the region containing the highest percentage of tumour cells (at least 50\%) was dissected. The paraffin was removed by xylene extraction, and the samples were subsequently lysed by proteinase K. DNA extraction was performed using a spin column procedure (QIAamp Tissue kit, Qiagen). Amplification of exon 19 E746_A750del (c.2236_2250del15 Deletion in frame, COSMICCatalogue of Somatic Mutations in Cancer http://www. sanger.ac.uk/search) and the L858R substitution in exon 21 (c.2573T>G) of the EGFR gene was based on RTPCR (Real-Time PCR kit, BioLink; CFX96 Real-Time System, Bio-Rad).

\section{Statistical analysis}

Quantitative variables were summarised as the means $\pm \mathrm{SD}$. Categorical variables were compared using the Chi-squared test (http://statpages.org/ctab2x2.html). Results were considered significant at the 0.05 level. Pearson's chi-squared, Yates' corrected chi-squared (when expected frequencies of less than 10 were found), and Fisher's exact (when expected frequencies of less than 5 were found) tests were taken into consideration for the assessment of genetic association.

\section{Results}

A total of 147 NSCLC cases were eligible for analysis. The mean age (SD) of the 147 patients was $59.8( \pm 8.9)$ years, age range $31-78$. Of the total, $31 \%$ of the patients had stage IV NSCLC with multiple metastasis, 22\% had stage III, 13\% had stage II, and 29\% had stage I. Adenocarcinoma was the most common histological type (65\%), followed by bronchioloalveolar carcinoma (17\%), large cell carcinoma (5\%), mixed adenocarcinoma (4\%), and NOS (9\%). The EGFR mutation status was identified before starting EGFR-TKI therapy. EGFR-TKI-sensitive mutations were detected in 28 of the 147 (19\%) patients. There were $19(13 \%)$ cases with mutations in exon 19 and 9 cases $(6 \%)$ in exon 21 . No patient had double mutations (exons 19 and 21). Mutations were more frequently observed in women $(42 \%, \mathrm{p}=0.000)$ than in men $(1 \%)$. A significantly higher incidence of EGFR mutations was observed in bronchioloalveolar carcinomas $(28 \%, \mathrm{p}=0.019)$ and in adenocarcinomas $(21 \%, \mathrm{p}=0.024)$ than in combined group (large cell carcinoma, mixed adenocarcinoma, NOS). The EGFR mutation rate was much higher in never-smokers than in smokers: $38 \%$ vs. $3 \%(\mathrm{p}=0.000)$. The frequency of EGFR mutation in the Kemerovo and Tomsk regions was $19 \%$.

\section{Discussion}

We assessed the frequency of EGFR mutations in West Siberian industrial regions (Tomsk and Kemerovo), where high rates of incidence and mortality due to lung cancer have been associated with environmental pollution. The Kemerovo region is dominated by metallurgy and the mining industry as well as mechanical engineering and chemical production (Mun et al., 2006). The Tomsk region is rich with natural resources, particularly oil, natural gas, 
ferrous and non-ferrous metals, peat, and underground waters (Kalinkin et al., 2014).

EGFR new line mutations were observed in 28 of 147 NSCLC patients (19\%). No significant difference in the frequency of EGFR mutation was observed between our study and previously performed studies by Russian scientists . Our results indicated that the frequency of EGFR mutations in NSCLC patients residing in West Siberia was similar to that observed in Western European and African-American patients (15-20\%) and significantly lower than in East Asian patients with NSCLC (30-60\%) (Leidner et al., 2009; Liam et al., 2014; Ahmed et al., 2014). The results of the present study regarding the relationship between the frequency of EGFR mutations and clinical pathological characteristics of NSCLC patients from West Siberia were consistent with worldwide findings. Mutations were more frequently observed in female patients, never-smokers, and in patients with bronchioloalveolar carcinomas and adenocarcinomas.

At the time of diagnosis, 15 of 28 EGFR-positive patients had stage IIIB-IV NSCLC with multiple metastases, and surgery was not an option for these patients. Thus, the findings of the present study may allow for expanding the pool of patients eligible for TKI treatment (gefitinib or erlotinib).

Although demographic and clinical factors such as East-Asian race, female gender, nonsmoking status, and adenocarcinoma were shown to be predictors of high EGFR mutation frequency (Yang et al., 2014), we carried out an investigation including male gender, smoking status, and other types of NSCLC. According to the recommendations given by Dara L. Aisner, 2012, EGFR mutation testing should include all cases with a component of adenocarcinomatous differentiation or those in which an adenocarcinomatous component cannot be excluded (Aisner et al., 2012). However, a large number of EGFRmutated cancer cells are required to predict a benefit from tyrosine kinase inhibitor treatment in NSCLC patients. In our study, only one patient with NSCLC-NOS had an EGFR mutation. The screening of EGFR mutations in NSCLC patients enables the clinician to provide adequate or alternative treatment not only for patients with adenocarcinoma but also for patients with other types of NSCLC. Recommendations given by Dara L. Aisner, 2012, also provide the possibility of excluding errors at the stage of diagnosis, partially based on phenomenon of intratumoral heterogeneity. Intratumoral heterogeneity is essential in lung cancer diagnosis which is usually made using biopsies or cytology specimens and also for EGFR mutation testing which is carried out without considering the tumor landscape. We have preliminary data (data not shown) in studying EGFR mutation status in different tumor clones of NSCLC by using Microtome (SLEE Medical GmbH, Germany), Microscopes Axio Scope A1 and Axio Star plus (Carl Zeiss, Germany), Laser microdissection with PALM MicroBeam (Carl Zeiss, Germany), 2200 TapeStation (Agilent Technologies, Inc., USA) (Denisov et al., 2014).

There were limitations in our study. First, in NSCLC patients, mutations in exons 21 (L861Q) and 18 (G719C, G719S, G719A) have clinically proven significance for TKI treatment and were not evaluated. Second, 121 NSCLC patients having no data on smoking history were excluded from the analysis. Heavy smokers are typically male, and most do not consider it necessary to report data on smoking history, preferring to conceal this information. In contrast, women diagnosed with NSCLC honestly report to their doctor whether they are never-smokers and never-drinkers.

Since 2012, the Tomsk Cancer Research Institute has begun to detect EGFR mutations in patients with NSCLC, taking into account the tumour histology, smoking status, and gender. EGFR mutation testing is now considered to be the standard of care for NSCLC patients treated at the Tomsk Cancer Research Institute. The median time elapsed between cancer diagnosis and the receipt of EGFR testing results is 5 working days.

The incorporation of molecular analysis of the EGFR gene into routine clinical practice will allow clinicians to provide personalised therapy, resulting in a significant increase in survival rates and improvement in life quality of advanced NSCLC patients.

\section{Acknowledgements}

This Research is partly supported by Federal Targeted Programmer for Research and Development in Priority Areas of Development of the Russian Scientific and Technological Complex for 2014-2020, «Development of molecular signatures for early detection of lung cancer» (№ 14.575.21.0064 from 05.08.2014). The study was carried out within the framework of the project "Improving the molecular genetic diagnosis of malignant tumours in the Russian Federation" under the patronage of RUSSCO (Professional Society of Oncologists Chemotherapeutics). The authors are grateful to Irina Demidova and Alexei Barinov from the Moscow City Oncology Hospital № 62 for their help in carrying out this project. This work was supported by a grant from the OPTEK Company (№22/2014/51-Nvs).

\section{References}

Ahmed ZA, Moatter T, Siddiqui A, et al (2014). Distribution of EGFR mutations commonly observed in primary lung adenocarcinomas in Pakistan as predictors for targeted therapy. Asian Pac J Cancer Prev, 15, 7125-8.

Aisner DL, Marshall CB (2012). Molecular pathology of nonsmall cell lung cancer: a practical guide. Am J Clin Pathol, 138, 332-46.

Cherdyntseva NV, Gervas PA, Litvyakov NV, et al (2010). Agerelated function of tumor suppressor gene TP53:contribution to cancer risk and progression. Exp Oncol, 32, 205-8.

Denisov EV, Litviakov NV, Zavyalova MV et al (2014). Intratumoral morphological heterogeneity of breast cancer: neoadjuvant chemotherapy efficiency and multidrug resistance gene expression. Sci Rep, 16, 4704-9.

Gridelli C, De Marinis F, Di Maio M, et al (2011). Gefitinib as first-line treatment for patients with advanced non-small-cell lung cancer with activating epidermal growth factor receptor mutation: implications for clinical practice and open issues. Lung Cancer, 72, 3-8.

Imianitov EN (2008). Molecular diagnosis in oncology. Mol Biol, 42, 772-85. 
Polina Gervas et al

Jemal A, Siegel R, Xu J, et al (2010). Cancer statistics, 2010. CA Cancer J Clin, 60, 277-300.

Kalinkin DE, Karpov AB, Takhauov RM et al. (2014). Mortality from cancer of the population living close to a large nuclear power plant. Vopr Onkol, 60, 41-6 (in Russian).

Leidner RS, Fu P, Clifford B, et al (2009). Genetic abnormalities of the EGFR pathway in African American patients with non-small-cell lung cancer. J Clin Oncol, 33, 5620-6.

Liam CK, Leow HR, How SH, et al (2014). Epidermal growth factor receptor mutations in non- small cell lung cancers in a multiethnic Malaysian patient population. Asian Pac J Cancer Prev, 15, 321-6.

Maemondo M, Inoue A, Kobayashi K, et al (2010). Gefitinib or chemotherapy for non-small-cell lung cancer with mutated EGFR. N Engl J Med, 25, 2380-8.

Mun SA, Larin SA, Brailovskii VV, et al (2006). Ambient air benz[a]pyrene and cancer morbidity in Kemerovo. Gig Sanit, 4, 28-30 (in Russian).

Ponomareva AA, Rykova EIu, Cherdyntseva NV et al. (2011). Molecular-genetic markers in lung cancer diagnostics. $\mathrm{Mol}$ Biol, 45, 203-17 (in Russian).

Yang ZM, Ding XP, Pen L, et al (2014). Analysis of CEA expression and EGFR mutation status in non-small cell lung cancers. Asian Pac J Cancer Prev, 15, 3451-5. 